\title{
BCG turns 100: its nontraditional uses against viruses, cancer, and immunologic diseases
}

\author{
Alok K. Singh, ${ }^{1}$ Mihai G. Netea, ${ }^{2,3}$ and William R. Bishai ${ }^{1}$ \\ 'Department of Medicine, Johns Hopkins School of Medicine, Baltimore, Maryland, USA. Department of Internal Medicine and Radboud Center for Infectious Diseases, Radboud University Medical Center, \\ Nijmegen, Netherlands. ${ }^{3}$ Department of Immunology and Metabolism, Life and Medical Sciences Institute, University of Bonn, Bonn, Cermany.
}

\begin{abstract}
First administered to a human subject as a tuberculosis (TB) vaccine on July 18, 1921, Bacillus Calmette-Guérin (BCC) has a long history of use for the prevention of TB and later the immunotherapy of bladder cancer. For TB prevention, BCC is given to infants born globally across over 180 countries and has been in use since the late 1920s. With about 352 million BCC doses procured annually and tens of billions of doses having been administered over the past century, it is estimated to be the most widely used vaccine in human history. While its roles for TB prevention and bladder cancer immunotherapy are widely appreciated, over the past century, BCC has been also studied for nontraditional purposes, which include (a) prevention of viral infections and nontuberculous mycobacterial infections, (b) cancer immunotherapy aside from bladder cancer, and (c) immunologic diseases, including multiple sclerosis, type 1 diabetes, and atopic diseases. The basis for these heterologous effects lies in the ability of BCC to alter immunologic set points via heterologous $\mathrm{T}$ cell immunity, as well as epigenetic and metabolomic changes in innate immune cells, a process called "trained immunity." In this Review, we provide an overview of what is known regarding the trained immunity mechanism of heterologous protection, and we describe the current knowledge base for these nontraditional uses of BCG.
\end{abstract}

\section{Historical perspective}

BCG is an attenuated strain of Mycobacterium bovis derived by serial passage. In conducting guinea pig virulence studies with Nocard's strain of M. bovis at the Pasteur Institute of Lille in 1908, Albert Calmette and Camille Guérin attempted to emulsify their strain by growth on a glycerinated-bile potato medium rather than grind clumps of bacilli in oil before animal inoculation (1). They noted a colony morphology change after 15 passages on this novel growth medium, and subsequently observed that this change correlated with reduced virulence in guinea pigs and calves (2). They went on to perform serial passage of the strain a total of 230 times over the next 13 years from 1908 to $1921(3,4)$. The end result was a strain that failed to produce progressive disease in at least six animal models and also conferred protection against challenge with virulent $M$. tuberculosis $(5,6)$. This strain was named Bacillus Calmette-Guérin (BCG; originally it was called Bacilli CalmetteGuérin) and was first administered as a vaccine on July 18, 1921, at the Charité Hospital in Paris by mouth to an infant boy whose mother had died of tuberculosis (TB) shortly after giving birth (7). By 1924, 664 infants had been vaccinated with few adverse effects, and protection was reported to be over $90 \%$ (8). These reports prompted requests from elsewhere around the globe, and

Conflict of interest: AKS and WRB hold patent applications on recombinant BCG (US16/147,916 to WRB; US16/638,943 to AKS and WRB). WRB is a shareholder in OncoSTING LLC. MGN holds patent applications for modulation of trained immunity (US18/61935 and US18/61939) and is scientific founder of Trained Therapeutix Discovery. Copyright: @ 2021, American Society for Clinical Investigation.

Reference information: J Clin Invest. 2021;131(11):e148291. https://doi.org/10.1172/JCl148291. as early as 1924 BCG was distributed widely (4, 9-11). The original BCG strain was distributed across the globe and was propagated on various culture media that led to diversification of the parent BCG into a number of genetically distinct BCG substrains. Over the decades from 1921 to 1960 (when BCG strains were first established as seed lots in long-term cryostorage), more than 14 substrains of BCG evolved with different mutations beyond those of the original strain developed by Calmette and Guérin $(12,13)$.

At present, BCG is recommended at birth in most countries for TB prevention. With about 352 million BCG doses procured annually $(14,15)$, it is possibly the most widely used vaccine in human history $(5,16)$. While its roles for TB prevention $(17,18)$ and bladder cancer immunotherapy (19) have been extensively described, here we present a non-systematic overview of nontraditional uses of BCG (Figure 1), a topic that has received only modest attention $(20,21)$.

\section{Trained immunity: a mechanism for BCG-mediated heterologous protection}

$\mathrm{BCG}$ is the only licensed vaccine for the prevention of TB, and per WHO recommendations it is routinely administered to infants after birth in TB-endemic regions (22). Numerous studies reveal that when given to neonates or school-age children, BCG confers significant protection against tuberculous meningitis and miliary TB in childhood (relative risk 0.04-0.12) and, to a lesser degree, pulmonary TB in childhood (relative risk 0.26-0.41) (see meta-analysis reported in ref. 23). It also offers more limited protection against TB in adulthood, especially adult pulmonary TB $(23,24)$. Observational and prospective cohort studies of neonatal $\mathrm{BCG}$ vaccination in West African countries show clear evidence that BCG reduces infant mortality risk by up to $30 \%-50 \%$ for up to 


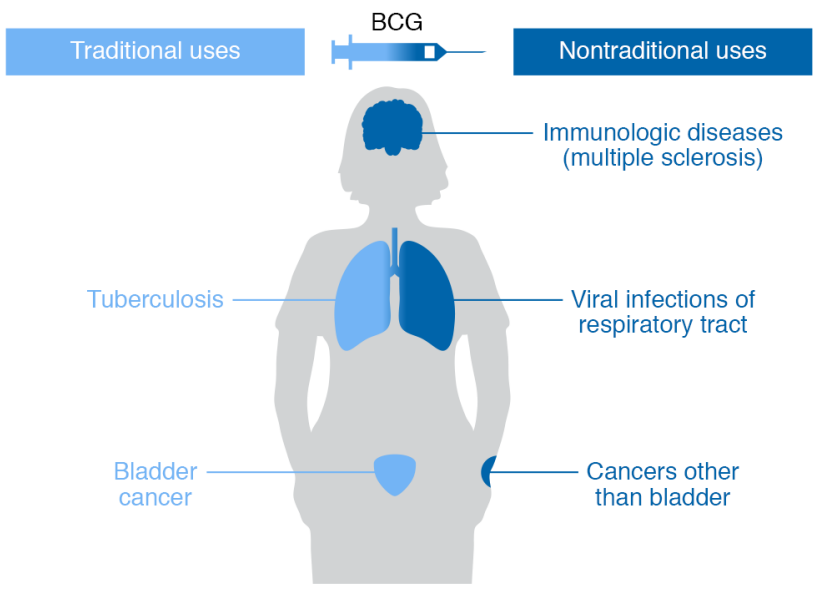

Figure 1. Schematic diagram depicting the traditional uses of BCG for protection against tuberculosis and immunotherapy for bladder cancer versus its nontraditional uses described in this Review for viral infections, cancer immunotherapy aside from bladder cancer, and immunologic diseases.

2 years of age, an effect so large that it suggested benefits of BCG beyond prevention of TB alone (25-27). Indeed, in observational and randomized prospective trials, neonatal BCG immunization of low-birth-weight infants was found to significantly improve survival for the first few months of life, mainly owing to prevention of non-TB-related lower respiratory tract infections $(28,29)$. Observations made in Malawi and Guinea-Bissau show clear evidence that BCG-mediated reduction of infant mortality is attributable to heterologous protective immunity against pediatric infections other than TB $(30,31)$. Similarly, a cluster sampling study of BCG immunization in infants in 33 countries revealed a reduction in the incidence of acute lower respiratory infection by $17 \%-37 \%$ (32). Further retrospective studies have revealed that BCG vaccination is associated with reductions in herpes simplex virus, respiratory syncytial virus, and influenza A $(33,34)$.

Importantly, not all studies have demonstrated heterologous protection against infections; a recent large prospective study of over 16,000 infants in Denmark is an example (35). While the exact reasons for such discrepancies are not known, heterologous protection by BCG appears to be more readily demonstrable in low-income countries, suggesting that disease prevalence, environmental antigenic exposures, and income discrepancy may be contributing factors.

The heterologous protection conferred by BCG is likely the result of two mechanisms that synergize to induce protection: heterologous T cell immunity, and "trained immunity," a term proposed to describe the ability of a first infection or antigenic exposure to develop an enhanced, nonspecific innate immune response that protects against a second infection independent of the adaptive immunity provided by $\mathrm{B}$ and $\mathrm{T}$ cells $(36,37)$. On the one hand, heterologous immunity that results in an improved response against pathogens other than the target microorganism was first reported by seminal studies by Mackaness (who called it "non-specific immunity") in the 1960s, showing that BCG can induce heterologous protection against other infections such as Listeria monocytogenes $(38,39)$. The role of $\mathrm{T}$ cells in this process has recently been underscored (40-42). On the other hand, trained immunity is mediated by an epigenetic, transcriptional, and functional reprogramming of innate immune cells such as monocytes, macrophages, or NK cells $(36,43,44)$. These initial events produce heterologous lymphocyte activation, resulting in enhanced proinflammatory cytokine production, macrophage activity, T cell responses, and antibody titers (41).

Early mechanistic studies of trained immunity of BCGinduced heterologous protection emerged from studies in the 1970s showing that BCG vaccination protected mice against $L$. monocytogenes $(45,46)$. Later, macrophages from BCG-primed animals were shown to be capable of increased killing of phagocytosed Candida albicans (47). Experimental validation of trained immunity in humans was done when monocytes from BCG-vaccinated adults showed elevated expression of cell surface markers of activation (CD11b and TLR4) and released more IL-1 $\beta$, IL-6, IFN- $\gamma$, and TNF- $\alpha$ in response to ex vivo Staphylococcus aureus or $C$. albicans infection for up to 3 months after vaccination as compared with monocytes that were isolated before vaccination from the same individuals (48). Durability of trained immunity responses was investigated in follow-up studies when monocytes from BCG-vaccinated individuals showed increased expression of CD14, TLR4, and mannose receptors as well as increased production of IFN- $\gamma$, IL-17, and IL-22 in response to heterologous stimulation for up to 1 year (41).

Epigenetic reprogramming in trained immunity. An important advance in understanding trained immunity was the demonstration that BCG training led to epigenetic modifications that alter gene expression patterns in innate immune cells. A study in 2012 revealed that BCG training is associated with NOD2-dependent epigenetic $\mathrm{H} 3 \mathrm{~K} 4$ trimethylation at gene promoters of surface activation markers and inflammatory cytokines (48). BCG training is also known to suppress H3K9 trimethylation, thus depressing the gene expression phenotypes in trained monocytes (ref. 49 and Figure 2).

Metabolic rewiring in trained immunity. Innate immune cells (monocytes and macrophages) show remarkable plasticity following immune stimulation by inducing metabolic pathways according to the need of an immune microenvironment. For example, inflammatory M1 macrophages depend largely on glycolysis and show impaired oxidative phosphorylation (OxPhos) and TCA cycle activity. In contrast, immunosuppressive M2 macrophages rely more on OxPhos, have increased $\beta$-oxidation as a result of fatty acid uptake, and their TCA cycle is active $(50,51)$. BCG-trained monocytes show a distinct pattern in the metabolic programming of the cell in which both glycolysis and OxPhos are increased (49, 52). A key outcome of this metabolic reprogramming is generation of metabolites that may act as cofactors for chromatin modifier enzymes. Indeed, pharmacologic and genetic modulations of rate-limiting glycolytic enzymes have been shown to inhibit BCG-induced epigenetic changes (H3K4me3 and H3K9me3), suggesting that increased glycolysis is an integral part of the immune training (49). Besides induction of glycolysis and increased lactate production, BCG-trained monocytes also show upregulation of the TCA cycle metabolites citrate, succinate, malate, fumarate, and 2-hydoxyglutarate as compared with naive macrophages, suggesting a possible role of glutaminolysis to supplement these specific metabolites $(53,54)$. In fact, the glutaminase inhibitor BPTES has been shown to reduce the efficiency of BCG-mediated trained 


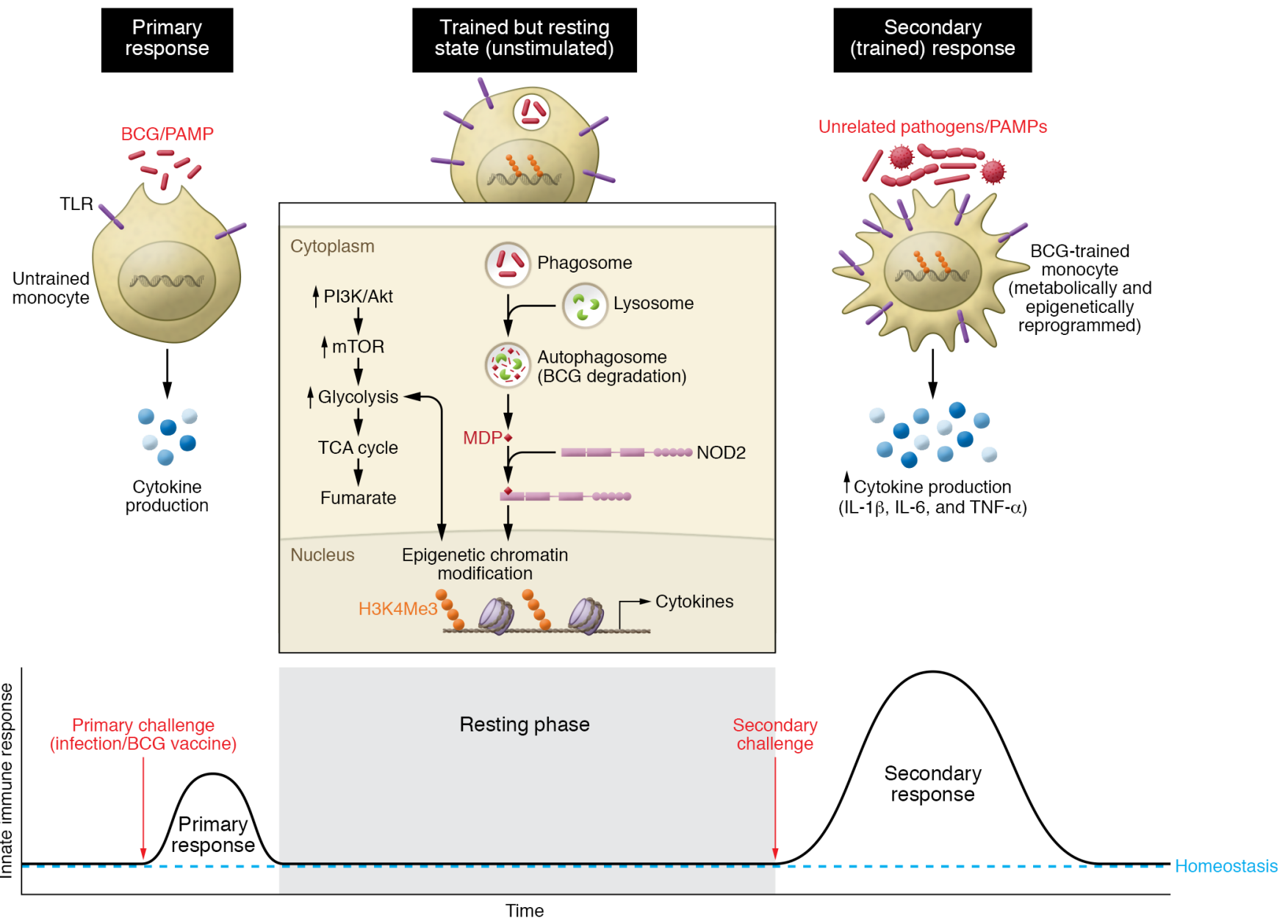

Figure 2. Epigenetic modifications and trained immunity. Schematic diagram depicting BCG-mediated trained immunity via epigenetic changes in myeloid cells that have encountered BCG. As shown, the primary challenge leads to "training" or reprogramming that includes durable, epigenetic modifications to chromatin, such as histone methylation (e.g., H3K4me3), that alter immune readiness. Following an encounter with a second immunologic challenge unrelated to BCC (secondary challenge), trained myeloid cells are capable of secondary immune responses of greater magnitude than those of untrained cells.

immunity in vitro and in experimental models. Importantly, metabolites such as $\mathrm{NAD}^{+}$and acetyl-CoA in trained monocytes serve as cofactors for histone deacetylases (HDACs) and histone acetyltransferases (HATs), respectively (55). Also, $\alpha$-ketoglutarate is an essential cofactor for several lysine- and cytosine-demethylating enzymes. Thus, shifts in metabolite levels in BCG-trained monocytes may be a plausible mechanism behind the integration of immunometabolic and epigenetic programs during trained immunity (refs. 53, 54, and Figure 3).

Heterologous protection mechanisms: unconventional $T$ cells. In addition to the previously mentioned role of NK cells in BCG-mediated heterologous protection, unconventional $\mathrm{T}$ cells such as innate lymphoid cells (ILCs) and mucosa-associated invariant $\mathrm{T}$ cells may also contribute to trained immune responses. In a recent murine study in which BCG was administered by the intradermal, subcutaneous, or intranasal route, ILCs were found in elevated numbers in lungs and lymph nodes and were found to be a significant source of IFN- $\gamma$ (56). Similarly, murine mucosa-associated invariant T cells demonstrated profound antimycobacterial responses and IFN- $\gamma$ production upon coculture with MR1-expressing macrophages that had been infected with BCG (57).
Heterologous protection mechanisms: role of $B C G$ antigens. While the precise pathogen-associated molecular patterns (PAMPs) involved in triggering BCG-mediated trained immunity remain unknown, it has been shown that BCG exposure leads to NOD2-dependent epigenetic changes and heterologous protection (48). In addition, it is known that the innate antigen for NOD2, muramyl dipeptide (MDP; a component of peptidoglycan in bacteria cell walls), is unique in mycobacteria ( $N$-glycolyl MDP) compared with other bacteria ( $N$-acetyl MDP) (58). Moreover, $N$-glycolyl MDP has been shown to be a more potent NOD2 stimulus than $N$-acetyl MDP (59). Thus, a unique $N$-glycolyl MDP may be implicated as a key antigenic trigger in BCG-mediated trained immunity.

Recent mechanistic work regarding the basis of trained immunity has implicated a BCG-mediated expansion of hematopoietic stem cells (HSCs) and multipotent progenitors in the bone marrow (60). These BCG-exposed marrow HSCs in turn generate epigenetically modified macrophages that demonstrate improved protection against $M$. tuberculosis challenge. A related study by the same group has addressed the question of whether mycobacteria all imprint trained immunity to the same degree and by similar mechanisms (61). Surprisingly, it was found that while the afore- 
Untrained innate immune cell

Basal metabolic activity

Less inflammatory

Energy source: TCA/OxPhos
BCG-trained innate immune cell

Increased metabolic activity

More inflammatory

Energy source: glycolysis

Epigenetically reprogrammed

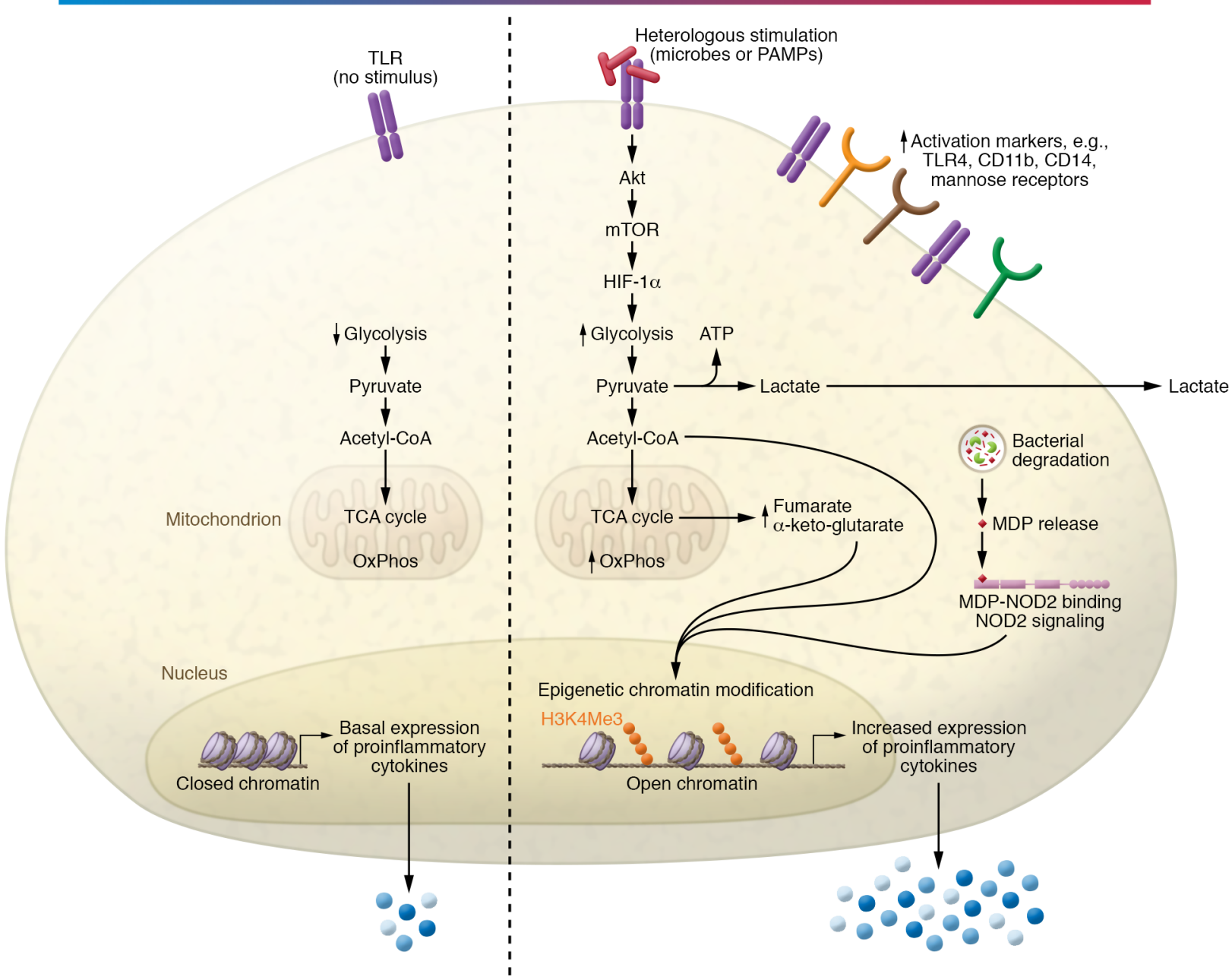

Figure 3. Metabolomic modifications and trained immunity. Schematic diagram depicting metabolomic changes associated with BCG-mediated trained immunity. Untrained innate immune cells (left) have relatively low levels of glycolysis and oxidative phosphorylation, and they express basal levels of proinflammatory cytokines. Following BCC training (right), metabolic activity in the glycolysis and oxidative phosphorylation pathways is increased, and upon immune restimulation (secondary challenge), immune responsiveness is elevated above levels seen with a primary challenge.

mentioned BCG-mediated marrow HSC expansion was associated with type II IFN release and improved macrophage immune training, M. tuberculosis in contrast impaired training and immunity by eliciting necroptosis of myeloid cell precursors in an environment of elevated type I IFN release. These findings suggest that not all mycobacteria elicit protective trained immune responses and that virulent mycobacteria seem to have evolved specific mechanisms to neutralize trained immunity as a means of escaping enhanced macrophage immune set points.

How can BCG be both immunostimulatory and immunotolerizing? As shown in Figure 1, BCG vaccination elicits proinflammatory, immunostimulatory responses against TB and bladder cancer but also appears to have immunotolerizing effects that play a role in its experimental uses against multiple sclerosis, type 1 diabetes, asthma, and atopic dermatitis. How BCG can elicit immunostimulatory respons- es in some and immunotolerizing effects in others remains largely unexplained. Hypotheses to account for such dichotomous responses include host factors such as (a) geographical factors (endemic mycobacterial exposure), (b) the presence of helminth infections, or (c) immunogenetic differences among human BCG recipients. Alternatively, microbial factors such as differences among BCG strains have also been proposed to explain dichotomous responses.

One potential answer to this conundrum comes from the consistent observation that BCG vaccination induces regulatory $\mathrm{T}$ cells (Tregs), a well-known cell population that induces immunologic tolerance $(62,63)$. Treg responses have been documented in both in children and adults receiving BCG for TB prevention (64-66) and in bladder cancer patients receiving BCG for cancer immunotherapy $(67,68)$. Indeed, this Treg response has been postulated as one of the limitations of BCG efficacy $(66,67)$. 
A recent small prospective study, in which 12 healthy, BCGnaive volunteers of European background were experimentally vaccinated with BCG, provided clear evidence of human dichotomous responses to BCG. While all vaccinees showed evidence of BCG uptake at 1 year, half of the subjects demonstrated proinflammatory polyfunctional $\mathrm{CD} 4^{+} \mathrm{T}$ cell responses and strong local skin inflammation at the BCG site. In contrast, the other half failed to mount polyfunctional $\mathrm{T}$ cells but rather showed a significant increase in $\mathrm{CD}^{+}$Tregs with minimal skin inflammation at the BCG site (69). Although the basis of these dichotomous responses remains unknown, the fact that they occurred in subjects from a non-mycobacteria-endemic area with few if any endogenous helminth infections with a single strain of BCG suggests that the basis for dichotomous responses to BCG may lie in the immunogenetic background of the recipient.

\section{BCG's impact on viral and nontuberculous mycobacterial infections}

Viral infections. In addition to the retrospective studies described in the previous section, BCG has also been studied prospectively for its protection against viral diseases and respiratory tract infections. For example, a randomized, placebo-controlled trial of 20 healthy adults who received BCG vaccination before H1N1 influenza vaccine, versus 20 who did not, found that prior BCG vaccination increased and accelerated the acquisition of functional anti-H1N1 antibody responses (70). In another randomized controlled trial, a prospective BCG vaccination study in adult volunteers was performed in which both placebo and BCG recipients were challenged 28 days later with the live attenuated yellow fever virus (YFV) vaccine and monitored for 90 days. BCG recipients had significantly lower YFV viremia 5 days after challenge and also displayed higher levels of IL-1 $\beta$ release, which correlated with epigenetic modifications. Despite the viremia reduction, BCG recipients showed no difference in YFV antibody titers at 90 days after challenge (71). This prospective virus challenge study clearly revealed the antiviral role of BCG-induced trained immunity and documented an epigenetic and cytokine release mechanistic basis for the effect. For efficacy against viral infections, BCG has been tested in three randomized controlled clinical trials (YFV, HPV, influenza A; refs. 7072), one case-control study (respiratory syncytial virus; ref. 73), and four case series (HPV and herpes simplex virus; refs. 74-77). Each showed a beneficial effect, and the preventive observations from the three randomized controlled trials strongly suggest causality for BCG against those viral infections. Comprehensive reviews of these studies have been published $(34,78)$.

$B C G$ vaccination for all-cause infections in elderly people. In another double-blind, randomized clinical trial (the phase III ACTIVATE trial; ClinicalTrials.gov NCT03296423) in patients 65 years of age or older, BCG versus placebo vaccination was administered at the time of hospital discharge, and patients were followed for 12 months for new infections. Compared with placebo, BCG vaccination significantly delayed the time to first infection (median 16 weeks compared with 11 weeks after placebo). Interestingly, the incidence of all-cause new infections was $42.3 \%$ (95\% CI 31.9\%-53.4\%) after placebo vaccination and 25.0\% (95\% CI $16.4 \%-36.1 \%$ ) after BCG vaccination. The impact of BCG was most profound against respiratory tract infections, including those of probable viral origin (hazard ratio $0.21, P=0.013$ ). This trial suggested that BCG vaccinations in elderly people are safe and can protect this population against respiratory infections (79). Importantly, however, no effects on gastrointestinal or urinary tract infections were observed, which warrants further investigation of the differential mechanisms involved in these infections.

BCG vaccination for COVID-19. During the past year, BCG emerged as a candidate vaccine for the prevention of infection with SARS-CoV-2 (80-82). Initial studies found that countries currently using BCG vaccination have lower SARS-CoV-2 incidence and death rates (83-87). One study, which sought to mitigate multiple confounding factors among countries (e.g., level of urbanization, population density, age structure, access to health, income, education, and stage of the COVID-19 epidemic) while assessing correlation between vaccination and COVID-19 mortality, found a significant correlation between the degree of BCG vaccination in a country and COVID-19 mortality (88). Another study using similar methods showed a correlation between BCG vaccination and daily rates of COVID-19 cases and deaths in the first 30-day period of a country's outbreak (83). Importantly, not all such correlation studies indicate a benefit of BCG in COVID-19 prevention; for example, a study that compared COVID-19 mortality by national BCG use patterns in early versus late 2020 did not find evidence of protection by BCG (89). A study performed in Israeli population cohorts before and after the discontinuation of BCG vaccination in the 1980s also failed to identify differences in COVID-19 incidence, arguing that BCG-induced protection is unlikely to be durable (90).

In contrast, in addition to these correlative studies, a retrospective observational study of a diverse cohort of 6679 health care workers in Los Angeles, California, demonstrated that a history of BCG vaccination was associated with reduced COVID-19related clinical symptoms $(P=0.017)$, as well as decreased seroprevalence of anti-SARS-CoV-2 IgG with an odds ratio (OR) of 0.76 (95\% CI 0.57-0.99; $P=0.048$ ) (91). Importantly, no association was found with meningococcal, pneumococcal, or influenza vaccination. While preliminary, these findings strengthen the argument that BCG may exert preventive efficacy and reduction in morbidity/mortality against SARS-CoV-2.

Importantly, prospective randomized clinical trials are necessary to make definitive conclusions regarding a potential effect of BCG vaccination against COVID-19. As of this writing, 22 clinical trials to test the efficacy of BCG are under way around the world and should produce more definitive answers on the role of BCG against COVID-19 (78-82, 92, 93).

$B C G$ for nontuberculous mycobacterial infections. In contrast to the other diseases described in this Review, nontuberculous mycobacterial (NTM) clearly share many common antigens with BCG, and hence BCG-mediated cross-immunity against NTM infections may not be based on heterologous or trained immunity, but rather upon broad antimycobacterial immunity conferred by BCG. We present this topic here because the use of BCG for prevention of NTM diseases remains nontraditional and experimental.

In developed regions such as the United States, Canada, and Western Europe where BCG is not used, rates of pulmonary NTM infections due to Mycobacterium avium, Mycobacterium abscessus, and related species have steadily increased in the past decades 
and are of particular concern for patients with cystic fibrosis (CF) or non-CF bronchiectasis $(94,95)$. While murine studies have clearly shown that BCG vaccination can protect against NTM infection (96), there is now renewed interest in whether BCG should be considered for the prevention of NTM infections, particularly in these high-risk subgroups. Human evidence for BCG-mediated protection against NTM infections comes from European nations with strong surveillance programs where BCG vaccination was suspended or terminated. In France, the mean incidence of culture-confirmed NTM cervical lymphadenitis sharply increased from 0.57 to 3.7 per 100,000 children per year after mandatory BCG vaccination was discontinued in 2007 (97). Similarly, in a large retrospective population-based study in Finland, where BCG policy changed from universal to selective vaccination strategy in 2006, childhood NTM infections increased drastically, with an incident rate ratio of 19.03 (95\% CI 8.8-41.07; $P<0.001$ ) (98). These population-based studies support the hypothesis that BCG offers cross-protection against NTM disease in humans. Recently, a prospective clinical trial in which five healthy people in St. Louis, Missouri, were given intramuscular BCG observed postvaccination increases among in vitro markers of immunity against NTM infections, including the induction of NTM-cross-reactive T cells (99).

Beyond pulmonary NTM infections caused by $M$. avium and related mycobacteria, BCG appears to confer a degree of protection against leprosy (Mycobacterium leprae) and Buruli ulcer (Mycobacterium ulcerans). At least three large cohort or case-control trials in Africa showed that BCG vaccination is associated with a $50 \%-80 \%$ reduction in the incidence of leprosy (100-102), and later, a randomized, controlled trial observed that a second BCG vaccination further improved leprosy prevention (103). Similarly, BCG vaccination was found to protect against Buruli ulcer in two large studies in Uganda (one a prospective observational study and the other a randomized controlled trial), although the duration of protection appears to be limited to only 6 to 12 months $(104,105)$.

\section{BCG immunotherapy for malignancies other than bladder}

History of BCG as immunotherapy for cancer. Microbe-based cancer immunotherapy dates back to the 19th century with the work of Busch and Fehleisen and of Coley using live or killed bacteria (106109). The role of mycobacteria in cancer interference was noted in 1928 by Pearl, who reported lower incidence of bladder cancer in patients with TB (110). Holmgren in Sweden was the first to use BCG for human cancer treatment in 1935 (9), and his studies were followed up in the 1960s by Vilasor and Mathé, who reported partial antitumor success $(111,112)$. In the 1950s, Lloyd Old demonstrated that mice infected with BCG had increased resistance to transplanted tumors (113). Later studies demonstrated antitumor effects of BCG against tumors in additional animal models (114, 115). Extensive reviews on the uses of BCG as an immunotherapy for a variety of human malignancies are available (116-118). These studies led to testing of intravesical BCG for bladder cancer and the seminal report by Morales et al. showing favorable results in nine bladder cancer patients treated with intravesical BCG (119). BCG is now standard therapy for non-muscle-invasive bladder cancer (reviewed in refs. 19, 120-122).
BCG to prevent cancer. BCG has also been studied for its preventive effects against cancer. Retrospective studies in the early 1970s by Davignon and later by Rosenthal revealed that neonatal BCG vaccination reduced incidence of acute leukemia (123-125), and a recent meta-analysis of BCG for childhood leukemia (12 studies) found protection with an OR of 0.73 (95\% CI 0.50-1.08) (126). BCG has also been studied for prevention against all-cause malignancies in large, long-term cohorts originally developed to study BCG against TB. A 1978 study in Puerto Rico of 50,634 vaccinated children and 31,586 controls did not observe fewer generalized malignancies at 18.8 years of follow-up (127). Similarly, a 1981 study of 16,913 BCG vaccinees and 17,854 controls from Georgia and Alabama who were 5 years old or greater found no generalized protective effect after 28 years (128). These long-term preventive studies were reviewed in 1990 (129). Recently, however, in contrast to the negative studies from four decades earlier, a 2019 paper reporting a 60-year retrospective analysis of 2963 BCG vaccinees in the United States showed a statistically significant reduction in lung cancer incidence (130).

$B C G$ for melanoma. From the mid-20th century until today, BCG has been used as an experimental intratumoral and systemic immunotherapy for a variety of solid tumors, including melanoma $(107,131)$. Indeed, the use of intralesional BCG remains listed in the National Comprehensive Cancer Network guidelines as an option for inoperable stage III melanoma (132).

Intralesional BCG was introduced in the early 1970s with a series of studies that showed complete regression in $15 \%-20 \%$ of recipients (133-135). In 1974, a larger study of intralesional therapy involving 36 patients with over 750 melanoma nodules found that $90 \%$ of the directly injected lesions regressed, as did $17 \%$ of the noninjected lesions (137). In 1993, an analysis of results pooled from 15 separate studies found that intralesional BCG in stage III melanoma resulted in complete responses in $19 \%$ and extended survival in 13\% (138). More recent studies of intralesional BCG have addressed its use in combination with topical immunotherapies such as imiquimod (a topical TLR7 agonist) and the experimental agent velimogene aliplasmid (a plasmid-lipid complex encoding HLA-B7 and $\beta_{2}$-microglobulin) (139, 140). Indeed, the use of imiquimod together with intralesional BCG was reported to yield high rates of complete regression in two small series $(141,142)$.

Systemic BCG - administered by skin scarification or by intradermal injection - has also been evaluated as an immune adjuvant for melanoma, although the results have been less compelling than for intralesional therapy. At least 19 trials in which BCG was given with either cytotoxic chemotherapeutics, cancer vaccines, or cytokine therapies have been reported, most with little difference conferred by the addition of BCG $(143,144)$. Nevertheless, certain large studies have suggested a benefit from systemic BCG. For example, one large study evaluating BCG and placebo versus BCG and the polyvalent melanoma cancer vaccine Canvaxin in 1160 stage III and 496 stage IV melanoma patients was stopped when the interim analysis showed no difference between groups (145). As all groups received intradermal BCG, the study was notable in that across study arms the overall projected 5 -year survival was $63 \%$ in stage III patients and $42 \%$ in stage IV patients, values significantly higher than those typically seen in melanoma. Long-term follow-up of the 
stage IV patients in the cohort, who all underwent surgical resection, failed to reveal a difference between groups but continued to show higher-than-expected survival rates in both arms (146).

More recently, intralesional BCG has been tested in combination with immune checkpoint inhibition. A dose escalation phase I study of intralesional BCG together with the anti-CTLA4 agent ipilimumab found that two of five patients developed immune-related adverse events, which included systemic toxicity, in conjunction with receiving the escalation doses of BCG (147). Prior to the onset of systemic toxicity, the study found increases in the repertoire of autoantibodies directed against both self- and cancer antigens.

$B C G$ for prostate cancer. Intradermal or intraprostatic BCG alone or in combination with chemotherapy was evaluated in prostate cancer in relatively small clinical trials primarily in the 1970 s and 1980s (148-151). In one of the largest series, involving 46 BCG recipients and 46 stage- and therapy-matched controls, reported in 1979, intradermal BCG recipients had prolonged survival compared with controls (37 vs. 21 months), and BCG-treated patients showed significant changes in complement levels and cutaneous hypersensitivity, suggesting an immune-mediated effect (152). In a similar study in 1982, 42 patients with advanced prostate cancer were randomized for either BCG adjuvant immunotherapy plus conventional therapy or conventional estrogen therapy; BCG recipients also showed prolonged survival compared with the control group (38 vs. 28 weeks, $P<0.05$ ) (151). Interestingly, quality-of-life indicators were significantly poorer in the BCG recipients. Despite these results showing a modest benefit of BCG in prostate cancer, there have been few subsequent studies in recent decades.

$B C G$ for lung cancer. Lung cancer immunotherapy before the 1990s remained focused on nonspecific immune stimulants including BCG (153). Lung cancer investigations were mainly focused on small cell lung cancer (SCLC) because of ease of treatment with minimal residual disease after curative chemotherapy and radiation (154). Some studies reported improved outcomes following intrapleural or intradermal BCG immunotherapy in patients receiving BCG and other modalities $(155,156)$, although this was not uniformly true. However, a 353-patient randomized phase III study in 1985 using a methanol extract of BCG as adjuvant therapy for SCLC failed to show an impact on overall survival (155), and in subsequent years few additional BCG studies in SCLC have been reported. However, very recently, a remarkable 60 -year follow-up study assessing the effect of BCG vaccination on cancer development suggested that childhood vaccination (single intradermal BCG vs. saline placebo) was associated with lower risk of lung cancer development (all types) in American Indian and Alaska Native populations (130).

\section{BCG for multiple sclerosis and other autoimmune diseases}

$B C G$ for multiple sclerosis. Multiple sclerosis (MS) is a chronic neuroinflammatory and neurodegenerative disease associated with immune-mediated demyelination of the central nervous system in which BCG has been tested as a disease modifier (157). Early animal studies showed that killed M. tuberculosis injections suppress demyelination in experimental autoimmune encephalomyelitis, an animal model of MS (158), prompting human studies. In a 1999 crossover study of 12 patients with relapsing-remitting MS who were followed with monthly MRI scans for 6 months, intracutaneous BCG administration revealed a 57\% reduction in MRI-active lesions and no adverse effects (159). In 2014, a double-blind, placebo-controlled study of 73 patients who had experienced a solitary first demyelinating event (clinically isolated syndrome) and were followed with monthly MRI scans for 6 months found that BCG recipients had significantly lower risk of developing new MRI lesions (relative risk 0.54, $P=0.03$ ). During a 5 -year follow-up period in which both arms received other disease-modifying therapies, 19 of $33(57.6 \%)$ of the BCG recipients remained MS free, while only 12 of $40(30 \%)$ were disease free in the control arm $(P=0.018)$; no major adverse events were observed with BCG treatment (160). One potential mechanistic explanation for the potential benefit of BCG is the glycolysis-inducing effect of BCG and the recent observation that patients with relapsing-remitting MS have impairments in glycolysis and mitochondrial respiration (161).

BCG for type I diabetes. Type 1 diabetes (T1D) is a chronic autoimmune disease wherein autoreactive $\mathrm{CD} 8^{+} \mathrm{T}$ cells destroy pancreatic $\beta$ cells as a result of increased targeting of islet cell autoantigens (162). The primary risk factor for $\beta$ cell autoimmunity is genetic, but environmental triggers are also implicated (163). BCG has been proposed as a beneficial environmental modifier of the immune system that can reduce the incidence of autoimmune diseases including T1D. In the autoimmune NOD (nonobese diabetic) murine model, either complete Freund's adjuvant (which contains heat-killed BCG) or BCG vaccination itself was observed to reduce diabetes development (164). In humans, neonatal BCG vaccination has been associated with reduction of autoantibodies associated with T1D such as GAD65 and I-A2 (165), and there are reports of preservation of $\beta$ cell function when BCG vaccination is administered soon after diabetes onset (166). Nevertheless, subsequent larger-scale studies in humans have failed to confirm that neonatal BCG vaccination (167) or BCG administered in newly diagnosed T1D (168, $169)$ reduces progression of diabetes or insulin requirements. Nevertheless, more recent studies have pointed out promising antidiabetic effects of BCG vaccination in patients with long-standing T1D, including a reduction of C-peptide (insulin fragment) (170) and TNF- $\alpha$-mediated selective death of autoreactive T cells and expansion of beneficial Tregs to restore immune balance (171).

Most recently, following the demonstration that BCG boosts glycolysis in BCG-trained human monocytes (49), the effects of BCG vaccination on reprogramming of immune metabolic state of T1D patients have garnered attention (172). In an 8-year human study involving 282 subjects with T1D, BCG vaccination was found to reduce hemoglobin $\mathrm{A}_{1 \mathrm{c}}$ levels to near normal levels, and, at least in immune cells, this correlated with a shift in glucose metabolism from OxPhos to aerobic glycolysis, a state of high glucose utilization, and reversal of ketosis (173). In the same study, CD $4^{+} \mathrm{T}$ cells from BCG-vaccinated adults with T1D showed a demethylation epigenetic mark in several Treg signature genes (FOXP3, TNFRSF18, IL2RA, $I K Z F 2, I K Z F 4$, and CTLA4) and a change in mRNA expression patterns likely to contribute to enhanced Treg generation and function. This induction of Tregs may be linked to the recently identified requirement of glycolysis for the generation of inducible Tregs (174). In contrast to much of the BCG literature, which shows proinflammatory effects, these observations suggest that BCG may also confer an immunotolerizing effect wherein Treg function is improved. 
BCG for asthma and other atopic diseases. Dozens of human studies have evaluated whether BCG plays a protective role in asthma and other atopic diseases, such as eczema/atopic dermatitis and allergic rhinoconjunctivitis. For example, a large case-control study of 751 Australian children 7 to 14 years of age observed a lower prevalence of current asthma among BCG recipients with a family history of rhinitis or eczema, again suggesting that BCG vaccination may provide Th1 stimulation and reduce IgE-responsiveness illness associated with allergic responses (175). For dermatologic skin diseases, a randomized, placebo-controlled trial with 121 predominantly White newborns with familial risk found a trend toward less eczema with a $P$ value of 0.07 (176). Similarly, in a large observational study of 400 children from Guinea-Bissau, early BCG vaccination was associated with a statistically significant decrease in atopic skin reactions to three dermatologic antigens, suggesting a BCG-mediated shift toward Th1 responsiveness (177). However, some studies have failed to show an impact of BCG vaccination on atopic responses (178).

To better understand BCG's effects in this arena, two systematic reviews have been conducted. The broader meta-analysis, which included 17 studies evaluating asthma as well as eczema/atopic dermatitis, allergic rhinoconjunctivitis, food allergies, and anaphylaxis, failed to find a protective effect of BCG for prevention of skin prick sensitization, eczema, or allergic rhinoconjunctivitis, but did observe protection against asthma with an OR of 0.73 (CI 0.89-1.28) (179). The second, which was restricted to asthma and included 23 studies, also found BCG to have a protective effect against asthma with an OR of 0.86 (CI 0.79-0.93) (180). Thus, there appears to be a consistent beneficial effect of BCG against asthma, while the evidence is less convincing for atopic skin diseases.

\section{Conclusions}

After 100 years of human use, BCG continues to be investigated against a variety of human diseases beyond its well-known roles in TB prevention and bladder cancer immunotherapy. In recent decades it has become clear that BCG modifies human immune cells both epigenetically and metabolically, and that these changes impart heterologous immune responsiveness to immunologic challenges unrelated to those of mycobacteria, a phenomenon that in innate immune cells has been termed "trained immunity." Of particular note is the observation that BCG-vaccinated individuals appear to have higher resistance to respiratory tract infections, and this has prompted a flurry of studies to determine if it may be useful against viral pandemics such as COVID-19 and for special populations at high risk of respiratory tract infection such as elderly people and CF patients.

While BCG clearly elicits proinflammatory responses, which contribute to its value as a bladder cancer immunotherapy, recent studies have demonstrated that its role in stimulating glycolysis may lead to an expansion of immunotolerizing Tregs; this may account for studies that reveal some efficacy of BCG in autoimmune diseases such as T1D and MS. In summary, beyond its wide use as a vaccine and immunotherapy during the past century, BCG continues to serve as a valuable biological probe that has helped elucidate fundamental properties of the human immune system.

\section{Acknowledgments}

The support of NIH grants AI152688, AI155346, and AI155602 (to AKS and WRB) and of European Research Council Advanced Grant 833247 and a Spinoza Grant of the Netherlands Organization for Scientific Research (to MGN) is gratefully acknowledged.

Address correspondence to: William R. Bishai, Department of Medicine, Johns Hopkins School of Medicine, 1550 Orleans Street, Baltimore, Maryland 21287, USA. Email: wbishai@jhmi.edu.
1. Calmette A, et al. Contribution à l'étude de la tuberculose expérimentale du cobaye. Ann Inst Pasteur. 1907;21:401-416.

2. Calmette A, Guérin C. Recherches expérimentales sur la défense de l'organisme contre l'infection tuberculeuse. Ann Inst Pasteur. 1911;25:625-641.

3. Rosenthal SR. BCG vaccination against tuberculosis. Am Rev Respir Dis. 1983;128(4):776.

4. Behr MA, Small PM. A historical and molecular phylogeny of BCG strains. Vaccine. 1999;17(7-8):915-922.

5. Tran V, et al. BCG vaccines. Microbiol Spectr. 2014;2(1):MGM2-0028-2013.

6. Guérin C, Rosenthal SR. The history of BCG: early history. In: Rosenthal SR, ed. BCG Vaccination Against Tuberculosis. J\&A Churchill; 1957:48-57.

7. Calmette A. Preventive vaccination against tuberculosis with BCG. Proc R Soc Med. 1931;24(11):1481-1490.

8. Sakula A. BCG: who were Calmette and Guérin? Thorax. 1983;38(11):806-812.

9. Crispen R. History of BCG and its substrains. Prog Clin Biol Res. 1989;310:35-50.

10. Luca S, Mihaescu T. History of BCG vaccine. Maedica (Bucur). 2013;8(1):53-58.
11. Calmette A. L'infection bacillaire et la tuberculose chez l'homme et chez les animaux. JAMA. 1923;80(17):1265.

12. Behr MA. Comparative genomics of BCG vaccines by whole-genome DNA microarray. Science. 1999;284(5419):1520-1523.

13. Pym AS, et al. Loss of RD1 contributed to the attenuation of the live tuberculosis vaccines Mycobacterium bovis BCG and Mycobacterium microti. Mol Microbiol. 2002;46(3):709-717.

14. Cernuschi T, et al. Bacillus Calmette-Guérin (BCG) vaccine: a global assessment of demand and supply balance. Vaccine. 2018;36(4):498-506.

15. Roy P, et al. Potential effect of age of BCG vaccination on global paediatric tuberculosis mortality: a modelling study. Lancet Glob Health. 2019;7(12):e1655-e1663.

16. Zwerling A, et al. The BCG World Atlas: a database of global BCG vaccination policies and practices. PLoS Med. 2011;8(3):e1001012.

17. Ottenhoff THM, Kaufmann SHE. Vaccines against tuberculosis: where are we and where do we need to go? PLoS Pathog. 2012;8(5):e1002607.

18. McShane H. Tuberculosis vaccines: beyond bacille Calmette-Guérin. Phil Trans R Soc B. 2011;366(1579):2782-2789.
19. Pettenati C, Ingersoll MA. Mechanisms of BCG immunotherapy and its outlook for bladder cancer. Nat Rev Urol. 2018;15(10):615-625.

20. Yamazaki-Nakashimada MA, et al. BCG: a vaccine with multiple faces. Hum Vaccin Immunother. 2020;16(8):1841-1850.

21. Kowalewicz-Kulbat M, Locht C. BCG and protection against inflammatory and auto-immune diseases. Expert Rev Vaccines. 2017;16(7):1-10.

22. World Health Organization. BCG vaccine: WHO position paper, February 2018 - recommendations. Vaccine. 2018;36(24):3408-3410.

23. Mangtani $P$, et al. Protection by BCG vaccine against tuberculosis: a systematic review of randomized controlled trials. Clin Infect Dis. 2014;58(4):470-480.

24. Andersen P, Doherty TM. The success and failure of BCG - implications for a novel tuberculosis vaccine. Nat Rev Microbiol. 2005;3(8):656-662.

25. Kristensen I, et al. Routine vaccinations and child survival: follow up study in Guinea-Bissau, West Africa. BMJ. 2000;321(7274):1435-1438.

26. Vaugelade J, et al. Non-specific effects of vaccination on child survival: prospective cohort study in Burkina Faso. BMJ. 2004;329(7478):1309.

27. Elguero E, et al. Non-specific effects of vaccina- 
tion on child survival? A prospective study in Senegal. Trop Med Int Health. 2005;10(10):956-960.

28. Aaby P, et al. Randomized trial of BCG vaccination at birth to low-birth-weight children: beneficial nonspecific effects in the neonatal period? J Infect Dis. 2011;204(2):245-252.

29. Moulton LH, et al. Evaluation of non-specific effects of infant immunizations on early infant mortality in a southern Indian population. Trop Med Int Health. 2005;10(10):947-955.

30. Aaby P, et al. Age-specific changes in the femalemale mortality ratio related to the pattern of vaccinations: an observational study from rural Gambia. Vaccine. 2006;24(22):4701-4708.

31. Biering-Sørensen S, et al. Small randomized trial among low-birth-weight children receiving bacillus Calmette-Guérin vaccination at first health center contact. Pediatr Infect Dis J. 2012;31(3):306-308.

32. Hollm-Delgado MG, et al. Acute lower respiratory infection among Bacille CalmetteGuérin (BCG)-vaccinated children. Pediatrics. 2014;133(1):e73-e81.

33. Butkeviciute E, et al. Heterologous effects of infant BCG vaccination: potential mechanisms of immunity. Future Microbiol. 2018;13(10):1193-1208.

34. Moorlag SJCFM, et al. Non-specific effects of $B C G$ vaccine on viral infections. Clin Microbiol Infect. 2019;25(12):1473-1478.

35. Kjærgaard J, et al. Nonspecific effect of BCG vaccination at birth on early childhood infections: a randomized, clinical multicenter trial. Pediatr Res. 2016;80(5):681-685.

36. Netea MG, et al. Trained immunity: a program of innate immune memory in health and disease. Science. 2016;352(6284):aaf1098.

37. O'Leary JG, et al. T cell- and B cell-independent adaptive immunity mediated by natural killer cells. Nat Immunol. 2006;7(5):507-516.

38. Mackaness GB. The immunological basis of acquired cellular resistance. JExp Med. 1964;120(1):105-120.

39. Mackaness GB. The influence of immunologically committed lymphoid cells on macrophage activity in vivo. J Exp Med.1969;129(5):973-992.

40. Kleinnijenhuis J, et al. BCG-induced trained immunity in NK cells: role for non-specific protection to infection. Clin Immunol. 2014;155(2):213-219.

41. Kleinnijenhuis J, et al. Long-lasting effects of BCG vaccination on both heterologous Th1/Th17 responses and innate trained immunity. JInnate Immun. 2014;6(2):152-158.

42. Boudreau JE, Hsu KC. Natural killer cell education and the response to infection and cancer therapy: stay tuned. Trends Immunol. 2018;39(3):222-239.

43. Netea MG, et al. Defining trained immunity and its role in health and disease. Nat Rev Immunol. 2020;20(6):375-388.

44. Divangahi $\mathrm{M}$, et al. Trained immunity, tolerance, priming and differentiation: distinct immunological processes. Nat Immunol. 2021;22(1):2-6.

45. Jespersen A. Acquired resistance of BCG-vaccinated red mice to infection with Listeria monocytogenes. Acta Pathol Microbiol Scand B. 1976;84B(5):265-272.

46. Ruitenberg EJ, Steerenberg PA. Stimulation of the non-specific resistance by Corynebacterium parvum and Bacillus calmette Guérin. Tijdschr Diergeneeskd. 1976;101(14):775-778.

47. Wout JW, et al. The role of BCG/PPD-activated macrophages in resistance against systemic candidiasis in mice. Scand J Immunol. 1992;36(5):713-720.

48. Kleinnijenhuis J, et al. Bacille Calmette-Guerin induces NOD2-dependent nonspecific protection from reinfection via epigenetic reprogramming of monocytes. Proc Natl Acad Sci U S A. 2012;109(43):17537-17542.

49. Arts RJW, et al. Immunometabolic pathways in BCG-induced trained immunity. Cell Rep. 2016;17(10):2562-2571.

50 . Viola A, et al. The metabolic signature of macrophage responses. Front Immunol. 2019;10:1462.

51. Artyomov MN, et al. Integrating immunometabolism and macrophage diversity. Semin Immunol. 2016;28(5):417-424.

52. Cheng SC, et al. mTOR- and HIF-1 $\alpha$-mediated aerobic glycolysis as metabolic basis for trained immunity. Science. 2014;345(6204):1250684.

53. Arts RJW, et al. Immunometabolic circuits in trained immunity. Semin Immunol. 2016;28(5):425-430.

54. Arts RJW, et al. Glutaminolysis and fumarate accumulation integrate immunometabolic and epigenetic programs in trained immunity. Cell Metab. 2016;24(6):807-819.

55. Yang XJ, Seto E. HATs and HDACs: from structure, function and regulation to novel strategies for therapy and prevention. Oncogene. 2007;26(37):5310-5318.

56. Steigler $\mathrm{P}$, et al. BCG vaccination drives accumulation and effector function of innate lymphoid cells in murine lungs. Immunol Cell Biol. 2018;96(4):379-389.

57. Chua WJ, et al. Polyclonal mucosa-associated invariant $\mathrm{T}$ cells have unique innate functions in bacterial infection. Infect Immun. 2012;80(9):3256-3267.

58. Behr MA, Divangahi M. Freund's adjuvant, NOD2 and mycobacteria. Curr Opin Microbiol. 2015;23:126-132.

59. Coulombe F, et al. Increased NOD2-mediated recognition of $\mathrm{N}$-glycolyl muramyl dipeptide. JExp Med.2009;206(8):1709-1716.

60. Kaufmann E, et al. BCG educates hematopoietic stem cells to generate protective innate immunity against tuberculosis. Cell. 2018;172(1-2):176-190.

61. Khan N, et al. M. tuberculosis reprograms hematopoietic stem cells to limit myelopoiesis and impair trained immunity. Cell. 2020;183(3):752-770.

62. Esensten JH, et al. Regulatory T-cell therapy for autoimmune and autoinflammatory diseases: the next frontier. J Allergy Clin Immunol. 2018;142(6):1710-1718.

63. Raffin C, et al. $\mathrm{T}_{\text {reg }}$ cell-based therapies: challenges and perspectives. Nat Rev Immunol. 2020;20(3):158-172.

64. Akkoc T, et al. Neonatal BCG vaccination induces $\mathrm{IL}-10$ production by $\mathrm{CD} 4+\mathrm{CD} 25+\mathrm{T}$ cells. Pediatr Allergy Immunol. 2010;21(7):1059-1063.

65. Li L, et al. The immune responses of central and effector memory BCG-specific CD4+ T cells in BCG-vaccinated PPD+ donors were modulated by Treg cells. Immunobiology. 2011;216(4):477-484.
66. Boer MC, et al. Regulatory T-cells at the interface between human host and pathogens in infectious diseases and vaccination. Front Immunol. 2015;6:217.

67. Chevalier MF, et al. Conventional and PD-L1-expressing regulatory $\mathrm{T}$ cells are enriched during BCG therapy and may limit its efficacy. Eur Urol. 2018;74(5):540-544.

68. Fenner A. BCG enriches $T_{\text {reg }}$ cells. Nat Rev Urol. 2018;15(10):591.

69. Boer MC, et al. Mycobacterium bovis BCG vaccination induces divergent proinflammatory or regulatory $\mathrm{T}$ cell responses in adults. Clin Vaccine Immunol. 2015;22(7):778-788.

70. Leentjens J, et al. BCG vaccination enhances the immunogenicity of subsequent influenza vaccination in healthy volunteers: a randomized, placebo-controlled pilot study. J Infect Dis. 2015;212(12):1930-1938.

71. Arts RJW, et al. BCG vaccination protects against experimental viral infection in humans through the induction of cytokines associated with trained immunity. Cell Host Microbe. 2018;23(1):89-100.e5.

72. Salem A, et al. Treatment of common and plane warts in children with topical viable Bacillus Calmette-Guerin. Pediatr Dermatol. 2013;30(1):60-63.

73. Stensballe LG, et al. Acute lower respiratory tract infections and respiratory syncytial virus in infants in Guinea-Bissau: a beneficial effect of BCG vaccination for girls community based case-control study. Vaccine. 2005;23(10):1251-1257.

74. Podder I, et al. Immunotherapy in viral warts with intradermal Bacillus Calmette-Guerin vaccine versus intradermal tuberculin purified protein derivative: a double-blind, randomized controlled trial comparing effectiveness and safety in a tertiary care center in Eastern India. Indian J Dermatol Venereol Leprol. 2017;83(3):411.

75. Daulatabad D, et al. BCG vaccine for immunotherapy in warts: is it really safe in a tuberculosis endemic area? Dermatol Ther. 2016;29(3):168-172.

76. Anderson FD, et al. Recurrent herpes genitalis. Treatment with Mycobacterium bovis (BCG). Obstet Gynecol. 1974;43(6):797-805.

77. Hippmann G, et al. [Nonspecific immune stimulation with BCG in Herpes simplex recidivans. Follow-up 5 to 10 years after BCG vaccination]. Wien Klin Wochenschr. 1992;104(7):200-204.

78. Sohrabi $Y$, et al. Trained immunity as a novel approach against COVID-19 with a focus on Bacillus Calmette-Guérin vaccine: mechanisms, challenges and perspectives. Clin Transl Immunology. 2020;9(12):e1228.

79. Giamarellos-Bourboulis EJ, et al. Activate: randomized clinical trial of BCG vaccination against infection in the elderly. Cell. 2020;183(2):315-323.

80. Netea MG, et al. BCG vaccination in healthcare providers and the protection against COVID-19. JClin Invest. 2021;131(2):e145545.

81. Netea MG, et al. Trained immunity: a tool for reducing susceptibility to and the severity of SARS-CoV-2 infection. Cell.2020;181(5):969-977.

82. Curtis N, et al. Considering BCG vaccination to reduce the impact of COVID-19. Lancet. 2020;395(10236):1545-1546.

83. Berg MK, et al. Mandated Bacillus CalmetteGuérin (BCG) vaccination predicts flattened 
curves for the spread of COVID-19. Sci Adv. 2020;6(32):eabc1463.

84. Gursel M, Gursel I. Is global BCG vaccination-induced trained immunity relevant to the progression of SARS-CoV-2 pandemic? Allergy. 2020;75(7):1815-1819.

85. Covián C, et al. Could BCG vaccination induce protective trained immunity for SARS-CoV-2? Front Immunol. 2020;11:970.

86. Miller A, et al. Correlation between universal $B C G$ vaccination policy and reduced morbidity and mortality for COVID-19: an epidemiological study [preprint]. https://doi.org/10.1101/ 2020.03.24.20042937. Posted on medRxiv September 14, 2020.

87. Shet A, et al. Differential COVID-19-attributable mortality and BCG vaccine use in countries [preprint]. https://doi.org/10.1101/2020.04.01.200 49478. Posted on medRxiv April 6, 2020.

88. Escobar LE, et al. BCG vaccine protection from severe coronavirus disease 2019 (COVID-19). Proc Natl Acad Sci U S A. 2020;117(30):17720-17726.

89. Lindestam Arlehamn CS, et al. Lack of evidence for BCG vaccine protection from severe COVID-19. Proc Natl Acad Sci U S A. 2020;117(41):25203-25204.

90. Hamiel U, et al. SARS-CoV-2 rates in BCG-vaccinated and unvaccinated young adults. JAMA. 2020;323(22):2340-2341.

91. Noval Rivas M, et al. BCG vaccination history associates with decreased SARS-CoV-2 seroprevalence across a diverse cohort of healthcare workers. JClin Invest. 2021;131(2):e145157.

92. O'Neill LAJ, Netea MG. BCG-induced trained immunity: can it offer protection against COVID19? Nat Rev Immunol. 2020;20(6):335-337.

93. Moorlag SJCFM, et al. Safety and COVID-19 symptoms in individuals recently vaccinated with BCG: a retrospective cohort study. Cell Rep Med. 2020;1(5):100073.

94. Ratnatunga $\mathrm{CN}$, et al. The rise of non-tuberculosis mycobacterial lung disease. Front Immunol. 2020;11:303.

95. Leung JM, Olivier KN. Nontuberculous mycobacteria: the changing epidemiology and treatment challenges in cystic fibrosis. Curr Opin Pulm Med. 2013;19(6):662-669.

96. Orme IM, Collins FM. Prophylactic effect in mice of BCG vaccination against nontuberculous mycobacterial infections. Tubercle. 1985;66(2):117-120.

97. Lacroix A, et al. Emergence of nontuberculous mycobacterial lymphadenitis in children after the discontinuation of mandatory Bacillus Calmette and Guérin immunization in France. Pediatr Infect Dis J. 2018;37(10):e257-e260.

98. Kontturi A, et al. Increase in childhood nontuberculous mycobacterial infections after Bacille Calmette-Guérin coverage drop: a nationwide, population-based retrospective study, Finland, 1995-2016. Clin Infect Dis. 2018;67(8):1256-1261.

99. Abate G, et al. BCG vaccination induces $M$. avium and $M$. abscessus cross-protective immunity. Front Immunol. 2019;10:234.

100.Pönnighaus JM, et al. Efficacy of BCG vaccine against leprosy and tuberculosis in northern Malawi. Lancet.1992;339(8794):636-639.

101.Stanley SJ, et al. BCG vaccination of children against leprosy in Uganda: final results. $J \mathrm{Hyg}$
(Lond). 1981;87(2):233-248.

102. Fine PE, et al. Protective efficacy of BCG against leprosy in Northern Malawi. Lancet. 1986;2(8505):499-502.

103. Randomised controlled trial of single BCG, repeated BCG, or combined BCG killed Mycobacterium leprae vaccine for prevention of leprosy tuberculosis in Malawi. Karonga Prevention Trial Group. Lancet. 1996;348(9019):17-24.

104.BCG vaccination against mycobacterium ulcerans infection (Buruli ulcer). First results of a trial in Uganda. Lancet. 1969;1(7586):111-115.

105. Smith PG, et al. The protective effect of BCG against Mycobacterium ulcerans disease: a controlled trial in an endemic area of Uganda. Trans R Soc Trop Med Hyg. 1976;70(5-6):449-457.

106. Oelschlaeger TA. Bacteria as tumor therapeutics? Bioeng Bugs. 2010;1(2):146-147.

107. Dobosz P, Dzieciątkowski T. The intriguing history of cancer immunotherapy. Front Immunol. 2019;10:2965.

108. Decker WK, et al. Cancer immunotherapy: historical perspective of a clinical revolution and emerging preclinical animal models. Front Immunol. 2017;8:829.

109. McCarthy EF. The toxins of William B. Coley and the treatment of bone and soft-tissue sarcomas. Iowa Orthop J. 2006;26:154-158.

110. Pearl R. On the pathological relations between cancer and tuberculosis. Exp Biol Med. 1928;26(1):73-75.

111. Villasor RP. The clinical use of BCG vaccine in stimulating host resistance to cancer. JPhilipp Med Assoc. 1965;41(9):619-632.

112. Mathé $\mathrm{G}$, et al. Active immunotherapy for acute lymphoblastic leukaemia. Lancet. 1969;1(7597):697-699.

113. Old LJ, et al. Effect of Bacillus Calmette-Guerin infection on transplanted tumours in the mouse. Nature. 1959;184(suppl 5):291-292.

114. Morales A. BCG: A throwback from the stone age of vaccines opened the path for bladder cancer immunotherapy. Can JUrol. 2017;24(3):8788-8793.

115. Coe JE, Feldman JD. Extracutaneous delayed hypersensitivity, particularly in the guinea-pig bladder. Immunology. 1966;10(2):127-136.

116. Nathanson L. Use of BCG in the treatment of human neoplasms: a review. Semin Oncol. 1974;1(4):337-350.

117. Bast RC, et al. BCG and cancer. N EnglJMed. 1974;290(26):1458-1469.

118. Hersh EM, et al. BCG as adjuvant immunotherapy for neoplasia. Annu Rev Med. 1977;28:489-515.

119. Morales A, et al. Intracavitary Bacillus Calmette-Guerin in the treatment of superficial bladder tumors. J Urol. 1976;116(2):180-183.

120. Redelman-Sidi G, et al. The mechanism of action of BCG therapy for bladder cancer-a current perspective. Nat Rev Urol. 2014;11(3):153-162.

121. Larsen ES, et al. Bacillus Calmette-Guérin immunotherapy for bladder cancer: a review of immunological aspects, clinical effects and BCG infections. APMIS. 2020;128(2):92-103.

122. Kates $M$, et al. Adaptive immune resistance to intravesical BCG in non-muscle invasive bladder cancer: implications for prospective BCG-unresponsive trials. Clin Cancer Res. 2020;26(4):882-891.

123. Davignon $L$, et al. $B C G$ vaccination and leukemia mortality. Lancet. 1970;2(7674):638

124. Davignon L, et al. BCG vaccination and leukaemia mortality. Lancet. 1971;1(7689):80-81.

125. Rosenthal SR, et al. BCG vaccination and leukemia mortality. JAMA. 1972;222(12):1543-1544.

126. Morra ME, et al. Early vaccination protects against childhood leukemia: a systematic review and meta-analysis. Sci Rep. 2017;7(1):15986.

127. Snider DE, et al. Efficacy of BCG vaccination in prevention of cancer: an update. J Natl Cancer Inst. 1978;60(4):785-788.

128. Kendrick MA, Comstock GW. BCG vaccination and the subsequent development of cancer in humans. J Natl Cancer Inst. 1981;66(3):431-437.

129. Grange JM, Stanford JL. BCG vaccination and cancer. Tubercle. 1990;71(1):61-64.

130. Usher NT, et al. Association of BCG vaccination in childhood with subsequent cancer diagnoses: a 60-year follow-up of a clinical trial. JAMA Netw Open. 2019;2(9):e1912014.

131. Sedighi M, et al. Therapeutic bacteria to combat cancer; current advances, challenges, and opportunities. Cancer Med. 2019;8(6):3167-3181.

132. Coit DG, et al. Cutaneous melanoma, version 2.2019, NCCN clinical practice guidelines in oncology. J Natl Compr Canc Netw. 2019;17(4):367-402.

133. Morton D, et al. Immunological factors which influence response to immunotherapy in malignant melanoma. Surgery. 1970;68(1):158-163.

134. Nathanson L. Regression of intradermal malignant melanoma after intralesional injection of Mycobacterium bovis strain BCG. Cancer Chemother Rep. 1972;56(5):659-665.

135. Seigler HF, et al. Non-specific and specific immunotherapy in patients with melanoma. Surgery. 1972;72(1):162-174.

136. Pinsky CM, et al. Treatment of malignant melanoma by intratumoral injection of BCG. Natl Cancer Inst Monogr. 1973;39:225-228.

137. Morton DL, et al. BCG immunotherapy of malignant melanoma: summary of a seven-year experience. Ann Surg. 1974;180(4):635-643.

138. Tan JK, Ho VC. Pooled analysis of the efficacy of bacille Calmette-Guerin (BCG) immunotherapy in malignant melanoma. J Dermatol Surg Oncol. 1993;19(11):985-990.

139. Bedikian AY, et al. A phase 2 study of high-dose Allovectin-7 in patients with advanced metastatic melanoma. Melanoma Res. 2010;20(3):218-226.

140. Sloot S, et al. Developments in intralesional therapy for metastatic melanoma. Cancer Control. 2016;23(1):12-20.

141. Kidner TB, et al. Combined intralesional bacille Calmette-Guérin (BCG) and topical imiquimod for in-transit melanoma. J Immunother. 2012;35(9):716-720.

142. Kibbi N, et al. Treatment of in-transit melanoma with intralesional bacillus Calmette-Guérin (BCG) and topical imiquimod 5\% cream: a report of 3 cases. J Immunother. 2015;38(9):371-375.

143. Kremenovic M, et al. Clinical and molecular insights into BCG immunotherapy for melanoma. J Intern Med. 2020;288(6):625-640.

144. Benitez MLR, et al. Mycobacterium bovis BCG in metastatic melanoma therapy. Appl Microbiol Biotechnol.2019;103(19):7903-7916.

145. Morton DL, et al. Prolonged survival of patients 
receiving active immunotherapy with Canvaxin therapeutic polyvalent vaccine after complete resection of melanoma metastatic to regional lymph nodes. Ann Surg. 2002;236(4):438-448.

146. Faries MB, et al. Long-term survival after complete surgical resection and adjuvant immunotherapy for distant melanoma metastases. Ann Surg Oncol. 2017;24(13):3991-4000.

147. Da Gama Duarte J, et al. Autoantibodies may predict immune-related toxicity: results from a phase I study of intralesional bacillus Calmette-Guérin followed by ipilimumab in patients with advanced metastatic melanoma. Front Immunol. 2018;9:411.

148. Guinan P, et al. Immunotherapy of prostate cancer: a review. Prostate. 1984;5(2):221-230.

149. Robinson MR, et al. Prostate carcinoma: intratumor BCG immunotherapy. Natl Cancer Inst Monogr. 1978;(49):351-353.

150. Guinan PD, et al. BCG adjuvant immunotherapy in carcinoma of the prostate: an interim report. Allergol Immunopathol (Madr). 1978;6(4):293-296.

151. Guinan P, et al. Bacillus Calmette-Guérin (BCG) adjuvant therapy in stage $\mathrm{D}$ prostate cancer. Urology. 1982;20(4):401-403.

152. Guinan P, et al. Adjuvant immunotherapy with bacillus Calmette-Guérin in prostatic cancer. Urology. 1979;14(6):561-565.

153. Raez LE, et al. Lung cancer immunotherapy. Clin Med Res. 2005;3(4):221-228.

154.Steven A, et al. Immunotherapy for lung cancer. Respirology. 2016;21(5):821-833.

155. Maurer LH, et al. Combined modality therapy with radiotherapy, chemotherapy, and immunotherapy in limited small-cell carcinoma of the lung: a phase III cancer and Leukemia Group B Study. JClin Oncol. 1985;3(7):969-976.

156. Yasumoto K, et al. Nonspecific adjuvant immunotherapy of lung cancer with cell wall skeleton of Mycobacterium bovis Bacillus Calmette-Guérin. Cancer Res. 1979;39(8):3262-3267.

157. Ristori $\mathrm{G}$, et al. Bridging the gap between vaccination with Bacille Calmette-Guérin (BCG) and immunological tolerance: the cases of type 1 diabetes and multiple sclerosis. Curr Opin Immunol. 2018;55:89-96.

158. Kies MW, Alvord EC. [Prevention of allergic encephalomyelitis by prior injection of adjuvants]. Nature. 1958;182(4642):1106.

159. Ristori G, et al. Use of bacille CalmetteGuèrin (BCG) in multiple sclerosis. Neurology. 1999;53(7):1588-1589.

160. Ristori G, et al. Effects of bacille Calmette-Guerin after the first demyelinating event in the CNS. Neurology. 2014;82(1):41-48.

161. La Rocca C, et al. Immunometabolic profiling of cells from patients with relapsing-remitting multiple sclerosis reveals an impairment in glycolysis and mitochondrial respiration. Metabolism. 2017;77:39-46.

162. In't Veld P. Insulitis in human type 1 diabetes: the quest for an elusive lesion. Islets. 2011;3(4):131-138.

163. Rewers M, Ludvigsson J. Environmental risk factors for type 1 diabetes. Lancet. 2016;387(10035):2340-2348.

164. Harada M, et al. Prevention of overt diabetes and insulitis in NOD mice by a single BCG vaccination. Diabetes Res Clin Pract. 1990;8(2):85-89.

165.Sanjeevi CB, et al. BCG vaccination and GAD65 and IA-2 autoantibodies in autoimmune diabetes in southern India. Ann N Y Acad Sci. 2002;958:293-296

166. Shehadeh N, et al. Effect of adjuvant therapy on development of diabetes in mouse and man. Lancet. 1994;343(8899):706-707.

167. Huppmann M, et al. Neonatal bacille Calmette-Guerin vaccination and type 1 diabetes. Diabetes Care. 2005;28(5):1204-1206.

168. Allen HF, et al. Effect of bacillus Calmette-Guerin vaccination on new-onset type 1 diabetes. A randomized clinical study. Diabetes Care. 1999;22(10):1703-1707.

169. Pozzilli P. BCG vaccine in insulin-dependent diabetes mellitus. IMDIAB Group. Lancet. 1997;349(9064):1520-1521.
170.Faustman DL, et al. Proof-of-concept, randomized, controlled clinical trial of bacillus-Calmette-Guerin for treatment of long-term type 1 diabetes. PLoS One. 2012;7(8):e41756.

171. Faustman DL. TNF, TNF inducers, and TNFR2 agonists: a new path to type 1 diabetes treatment. Diabetes Metab Res Rev. 2018;34(1):10.1002/dmrr.2941.

172.Faustman DL. Benefits of BCG-induced metabolic switch from oxidative phosphorylation to aerobic glycolysis in autoimmune and nervous system diseases. J Intern Med. 2020;288(6):641-650.

173. Kühtreiber WM, et al. Long-term reduction in hyperglycemia in advanced type 1 diabetes: the value of induced aerobic glycolysis with BCG vaccinations. NPJ Vaccines. 2018;3:23.

174. De Rosa V, et al. Glycolysis controls the induction of human regulatory $\mathrm{T}$ cells by modulating the expression of FOXP3 exon 2 splicing variants. Nat Immunol. 2015;16(11):1174-1184.

175. Marks GB, et al. The effect of neonatal BCG vaccination on atopy and asthma at age 7 to 14 years: an historical cohort study in a community with a very low prevalence of tuberculosis infection and a high prevalence of atopic disease. JAllergy Clin Immunol. 2003;111(3):541-549.

176. Steenhuis TJ, et al. Bacille-Calmette-Guerin vaccination and the development of allergic disease in children: a randomized, prospective, single-blind study. Clin Exp Allergy. 2008;38(1):79-85.

177. Aaby P, et al. Early BCG vaccination and reduction in atopy in Guinea-Bissau. Clin Exp Allergy. 2000;30(5):644-650.

178. Alm JS, et al. Early BCG vaccination and development of atopy. Lancet. 1997;350(9075):400-403.

179. Arnoldussen DL, et al. BCG vaccination and allergy: a systematic review and meta-analysis. J Allergy Clin Immunol. 2011;127(1):246-253.

180.El-Zein M, et al. Does BCG vaccination protect against the development of childhood asthma? A systematic review and meta-analysis of epidemiological studies. Int JEpidemiol. 2010;39(2):469-486. 